\title{
Violencias contra mujeres en Veracruz
}

\section{Estela Casados González ${ }^{1}$}

A la Maestra Guadalupe Mora Palacios Asesinada en su hogar, en pleno corazón de Xalapa, el 10 de noviembre de 2016. Sus asesinos están libres.

RESUMEN: En Veracruz la segunda década del siglo XXI ha estado marcada por la inseguridad y la violencia. Específicamente, las agresiones hacia las mujeres de esta entidad se incrementaron gravemente. Este artículo expondrá un análisis comparativo sobre lo sucedido en materia de violencias contra mujeres en la entidad en el primer semestre de 2016 y el mismo periodo correspondiente a 2017.

Palabras Clave: violencias, mujeres, desaparición, feminicidio, observatorio.

ABSTRACT: In Veracruz the second decade of the 21st century has been marked by insecurity and violence. Specifically, aggressions towards women in this state increased seriously. This article will present a comparative analysis of what happened in the subject of violence against women in the first half of 2016 and the corresponding period of 2017.

Keywords: violence, women, missing, femicide, observatory.

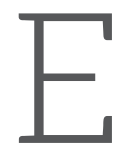
desarrollo humano de las mujeres está gravemente comprometido cuando son víctimas de cualquier tipo de violencia. La pérdida de su autonomía y de sus vidas es

1 Docente Investigadora en la Facultad de Antropología. Coordina la Maestría en Estudios de Género en el Centro de Estudios de Género la Universidad Veracruzana-CEGUV. Responsable del Observatorio Universitario de Violencias contra Mujeres en el estado de Veracruz. Nivel 1 del Sistema Nacional de Investigadores (SNI). Profesora con Perfil Deseable del Programa para el Desarrollo Profesional Docente(PRODEP).Contacto: ecasados@uv.mx 
la muestra más contundente de un fenómeno social que ha sido normalizado y justificado a lo largo de la historia de la humanidad, pero que en las últimas décadas se ha recrudecido duramente.

La violencia hacia las mujeres constituye una grave violación hacia los derechos humanos. Merma la seguridad y la democracia que debe garantizar un Estado. Bajo esta falta, Veracruz inició el siglo XXI exhibiendo la vulnerabilidad de la ciudadanía ante el fortalecimiento del crimen organizado. De inmediato, esto se reflejó en los cuerpos femeninos. Fueron el termómetro y lugar común en donde se exhibió su fortaleza.

Simultáneamente, viejos actores conocidos por el maltrato que prodigan a sus contrapartes femeninas, incrementaron la violencia que tradicionalmente ejercían tanto en el ámbito público como el privado. Los compañeros íntimos, exparejas, familiares, vecinos, compañeros de trabajo, de la escuela o el desconocido que camina la misma calle, encarnan a los agresores quienes, ante la percepción de impunidad que sobreviene cuando se ataca a las mujeres, han intensificado las agresiones.

El objetivo de este artículo es presentar algunos datos que permitan comprender y analizar las agresiones hacia las veracruzanas, que en su más elevada escala de violencia se traducen en feminicidio y homicidio de mujeres. Es a partir de los datos recabados por el proyecto Asesinatos de mujeres y niñas por razón de género. Feminicidio en la entidad veracruzana, que conoceremos las cifras de agresiones en dos periodos inmediatos.

Para tal efecto, se realizará un ejercicio comparativo entre la prevalencia de agresiones a mujeres durante el primer semestre del año 2016 y el correspondiente a 2017; ello con la finalidad de medir la dinámica que se ha establecido en los últimos tiempos. Se retomarán tres ejes importantes de violencia: feminicidio y homicidios de mujeres, desaparición forzada de veracruzanas, así como los diferentes tipos de violencia a los que se ven expuestas.

Para el registro y análisis de datos, el proyecto utiliza la metodología feminista, tomando como punto de partida a la teoría feminista y los estudios de género, que también guían las siguientes páginas. Cabe mencionar que las cifras recabadas obedecen a un monitoreo de medios de comunicación locales (impresos y portales informativos), así como redes sociales. Por lo que se recuperarán estos números.

En este artículo se utilizará la expresión violencia o violencias contra las mujeres y no violencia de género, ello con el propósito de resaltar que existen violencias específicas que tienen un carácter sexista y discriminatorio que se diluyen o invisibilizan al utilizar la categoría género, a pesar del carácter relacional, contextual e histórico de la misma. Es fundamental resaltar que la violación a los derechos humanos de las mujeres acontece en contextos influenciados por una cultura misógina que sega su seguridad, autonomía, sino es que sus vidas.

Sobre el tema, un debate recurrente en América Latina gira en torno a la conceptualización de la violencia hacia las mujeres como violencia de género o en el contexto de la violencia doméstica o intrafamiliar. Cuando se diseñaron las primeras herramientas legales al respecto en países como Argentina, Chile, Perú, entre otros más, se adoptaron leyes que se enmarcaban en el contexto de violencia familiar desde una perspectiva que pretendía ser integral. Es decir, se hacía en referencia tanto a las mujeres como a los hombres (Almerás y Calderón, 2012: 23)

Fue gracias al trabajo teórico y político feminista que se comenzó a plantear la necesidad de construir un andamiaje legal que conside-

\footnotetext{
2 Dicho proyecto se realiza en la Licenciatura en Antropología Social, en la Facultad de Antropología de la Universidad Veracruzana. SIREI-DGI 231222011681

3 Para profundizar en la teoría y metodología feministas, entre otros textos, se recomienda remitirse a Martha Patricia Castañeda Salgado (2006), "La antropología feminista hoy: algunos énfasis claves"; Itzel Sosa Sánchez (2008), "Feminismo y Ciencias Sociales"; Margaret Bullen (2012), "Antropología feminista, antropología aplicada. Encuentros y desencuentros"; Norma Blazquez Graf, Fátima Flores Palacios, Maribel Ríos Everardo (Coordinadoras) (2012), Investigación feminista: epistemología, metodología y representaciones sociales, México, UNAM; entre otros.

4 Se han consultado 57 medios impresos, portales informativos y espacios informativos de internet..
} 
rara las formas específicas en que la violencia afecta a las mujeres. A través de distintos instrumentos jurídicos internacionales, México se ha comprometido a impulsar programas y políticas que tengan como objetivo prevenir, atender y sancionar la violencia contra las mujeres.

Así, la convención Interamericana para la Prevención, Sanción y Erradicación de la Violencia contra la Mujer (popularmente conocida como Belém Do Pará) ha vinculado a países de la Organización de Estados Americanos (OEA), los cuales la han ratificado o se han adherido a ella. El 19 de enero de 1999, México decretó la promulgación de dicha Convención en el Diario Oficial, para posteriormente decretar la Ley General de Acceso de las Mujeres a una Vida Libre de Violencia, el 1 de febrero de 2007 (INMUJERES, 2009: 5). La Ley número 235 de Acceso de las Mujeres a una Vida Libre de Violencia para el estado de Veracruz de Ignacio de la Llave fue publicada en la Gaceta Oficial el 28 de febrero de 2008.

En el presente artículo se entiende por violencia contra las mujeres como "todo acto de violencia basado en la pertenencia al sexo femenino que tenga o pueda tener como resultado un daño o sufrimiento físico, sexual o sicológico para la mujer, así como las amenazas de tales actos, la coacción o la privación arbitraria de la libertad, tanto si se producen en la vida pública o privada" (ONU, 1993:2).

Cuando se utilice el término en plural, violencias contra mujeres, se estará haciendo referencia a las diferentes manifestaciones que la conforman: homicidios de mujeres, feminicidios, desaparición femenina forzada y agresiones contra mujeres.

\section{Pobreza e inseguridad en Veracruz y su reper- cusión en las violencias hacia las mujeres}

El recrudecimiento de la inseguridad ha to- mado por sorpresa a la población veracruzana. Sus esquirlas han diezmado la dinámica y desarrollo económico, el derecho al libre tránsito por el espacio público, así como la vida de un número aún no determinado de habitantes del estado. ¿Cómo ha afectado todo ello a las ciudadanas que habitamos este territorio?

Podemos hilvanar algunas respuestas si observamos brevemente el panorama que ha privado en Veracruz en los últimos años. Este ejercicio nos permitirá entender los alcances de la violencia para la población en general y para las mujeres en particular.

Un punto de partida es concentrarnos en la calidad de vida de sus habitantes y analizar cómo es que ha repercutido estructuralmente. Tal como lo establecieron Hipólito Rodríguez y Gilberto Cházaro (2013: 23), se ha observado que no es la pobreza la que genera situaciones de vulnerabilidad para que se incurra en prácticas delictivas, sino que éstas encuentran más posibilidades de desarrollarse en ámbitos donde es aguda la desigualdad.

En este sentido, el Programa de las Naciones Unidas para el Desarrollo (PNUD, 2015:6), establece que el estado de Veracruz se ubica en el sitio número cinco de entre las entidades federativas con menor índice de desarrollo humano en México. En el documento Índice de Desarrollo Humano para las entidades federativas, México 2015. Avance continuo, diferencias persistentes, el PNUD concluye que esta situación se deriva de la falta de transparencia en el uso de recursos públicos que evidencie, si es el caso, su uso adecuado y oportuno (ibid, p. 18).

Las conclusiones del PNUD forman parte de un rompecabezas de datos que permiten observar a fondo la situación estatal desde los primeros años del siglo XXI. Así tenemos que de los 20 municipios con mayor desigualdad en el país, ocho se encuentran en Veracruz (Rodríguez y Cházaro, 2013:25).

\footnotetext{
5 Agresiones contra mujeres hace referencia a los tipos y modalidades de violencia hacia las mujeres definidas en la Ley de Acceso de las Mujeres a una vida Libre de Violencia para el estado de Veracruz de Ignacio de la Llave.

6 La media nacional es de 0.746, siendo que Ciudad de México tenía el 0.830. El estado de Veracruz se ubicaba en 0.713 (PNUD, 2015: 9).

7 Xalapa, Fortín, Boca del Río, Mariano Escobedo, Minatitlán, Coatzacoalcos, Nogales y Tuxpan; Fuente: Municipios con mayor y menor desigualdad de ingreso, 2005, PNUD (2007, Índice de desarrollo humano municipal en México 20002005, p. 19; citado por Rodríguez y Cházaro, 2013).
} 
A esto se suma la información aportada por el Consejo Nacional de Evaluación de la Política de Desarrollo Social (CONEVAL) con relación a las seis carencias sociales que conforman la medición de pobreza. Para el año 2015 en Veracruz se encontraron datos por demás alarmantes:

El 26\% de la población presentaba rezago educativo, 19.2\% carecía de servicios de salud y el 19.5\% no contaba con acceso a calidad y espacios en la vivienda. Es de llamar la atención que el 30.1\% de quienes habitaban la entidad veracruzana no contaban con acceso a la alimentación. El 68.3\% carecía de acceso a seguridad social y el $40.9 \%$ no contaba con servicios básicos de vivienda (CONEVAL, 2017a).

Lo anterior ubicó a Veracruz entre las cinco entidades de la república mexicana con mayor rezago educativo, menor acceso a vivienda de calidad y a servicios básicos en la misma, así como menor acceso a la alimentación. Otro dato sobresaliente que expone CONEVAL (2017b) es que las entidades federativas con mayores disminuciones en el ingreso laboral real de sus habitantes en el periodo 2010 a 2016, son Veracruz, con el -14.3\%; Morelos, -13.6\%; Tabasco, -9.9\%; Guerrero, -7.3\%; Nuevo León, -6.5\%.

Durante 2014, este mismo organismo nacional, ante los datos abrumadores de medición de pobreza y disminución de ingreso laboral, estableció que las mexicanas pobres se ocupan con mayor frecuencia en jornadas parciales de trabajo, lo que trae consigo remuneraciones menores. Incluso si cuentan con la misma escolaridad que sus compañeros de trabajo, su paga será menor. Paralelamente, tienen mayor dependencia de los recursos provenientes de programas sociales (CONEVAL, 2014: 1).

Tan solo estos breves datos permiten observar que la información y el registro estadístico disponible indican que "la delincuencia y la violencia suelen desplegarse en espacios geográficos donde es mayor la desigualdad socioe- conómica y el deterioro de las instituciones de justicia" (Rodríguez y Cházaro, 2013: 23). Pobreza y desigualdad pueden convertirse en factores detonantes de la inseguridad. Así la distribución de la pobreza en un espacio geográfico aporta elementos que permiten hacer más preciso el diagnóstico de las circunstancias que colocan a amplias capas de la población en situaciones de riesgo frente a la inseguridad (ibid, p. 49).

Un elemento importante que no debemos dejar de lado es que en los últimos años los recursos públicos estatales fueron saqueados y las carencias sociales reflejadas por el CONEVAL son clara muestra de ello. Las condiciones de pobreza y desigualdad que se fueron creando en la primera década del siglo XXI favorecieron los contextos de violencia hacia las mujeres, sobre todo para las más pobres. Sin embargo, esto aún no ha tocado fondo. La segunda década de este siglo también está mostrando niveles insospechados.

Además de la pobreza y desigualdad, hay otros factores más que abonan a la violencia contra las veracruzanas: las instituciones que tienen la tarea de procurar justicia se encuentran rebasadas ante los altos índices de violencia; el desinterés de los tres poderes de gobierno para concretar de manera efectiva la legislación para proteger la vida de las mujeres; el mensaje de impunidad al no procurar justicia ante el caos de violencia contra las mujeres; el desmantelamiento paulatino de instancias para la tranversalización de la perspectiva de género, tal como es el caso del Instituto Veracruzano de las Mujeres.

De acuerdo con información oficial presentada por el estado de Veracruz, del año 2000 al 2014 se presentaron 1196 casos de homicidios dolosos de mujeres y niñas. La edad de las mujeres oscilaba, principalmente, entre los 21 y 30 años (190 víctimas mortales). En el rango de 31 y 40 años se registraron a 150 víctimas; entre

\footnotetext{
8 En un esfuerzo por entender la situación que vive la población en suelo veracruzano, algunos autores han acuñado el concepto bolsones de pobreza, en referencia a áreas geográficas donde se concentran hogares y personas con rezagos importantes que ven restringidas sus opciones ante la dificultad de acceder a bienes y servicios (ibid, p. 44 y 48).

9 Víctimas que acuden a las organizaciones de la sociedad civil o que se atreven a hacer declaraciones en eventos públicos sobre sus experiencias al acudir como usuarias a la Fiscalía Especializada para la atención de delitos contra mujeres, coinciden en que las fiscales les recomiendan desistir de sus denuncias. Les informan que sus casos difícilmente prosperarán y que sus agresores no pisarán la cárcel.
} 
los 11 y 20 años de edad, se establecieron 144 víctimas. Asimismo, en el rango que va de los 41 a 50 años, fueron registradas 123 víctimas. Finalmente, de 51 a 60 años, 87 víctimas (CONAVIM, 2015: 26).

En 2011 se tipificó el delito de feminicidio en el Código Penal de Veracruz. Datos oficiales establecen que en 2012 las autoridades dieron a conocer al gobierno federal que se registraron once feminicidios; en 2013, 52 casos; en 2014, fueron 43. Es decir, un total de 106.

Para el año 2015, el Grupo de Trabajo que se constituyó con motivo de la solicitud de Alerta de Violencia de Género contra las Mujeres en el estado de Veracruz por feminicidio, en su informe de trabajo señaló que "en el estado de Veracruz existe un contexto de violencia estructural que se deriva de la situación de alta marginación y pobreza, la ubicación geográfica de la entidad, los flujos migratorios a los está sujeta y la exclusión de las diversas comunidades indígenas que habitan el estado" (CONAVIM, 2015: 67).

Asimismo, el informe señala que Veracruz presenta diversos fenómenos alarmantes:

1. Altas tasas de reincidencia de actos de violencia, lo que muestra una situación de violencia constante en la vida de muchas mujeres de a entidad.

2. Tendencia creciente de los egresos hospitalarios femeninos relacionados con violencia.

3. Incremento de desapariciones de mujeres. Siendo menos del 50\% el número de mujeres localizadas.

4. La maternidad de menores de 15 años es mayor que el promedio nacional (ibid, p. 68).

\section{Violencias contra veracruzanas}

En este apartado se expondrá el número de casos correspondiente a los reportes de violencias contra mujeres emitidos por medios de comunicación locales, así como aquellos sobre desaparición forzada de veracruzanas, feminicidio y homicidio. Será a partir de un ejercicio comparativo que se analizará el primer semestre de 2016 y el mismo periodo correspondiente a 2017. Esta estrategia tiene la intención de observar la situación que prevalecía antes de que se implementara la declaratoria de Alerta de Violencia de Género y los posibles cambios efectuados una vez que se echara a andar este mecanismo federal.

No olvidemos que después de un largo proceso que duró más de un año, diez organizaciones de la sociedad civil consiguieron que el gobierno federal emitiera la Declaratoria de Alerta de Violencia de Género contra las mujeres para el estado de Veracruz por el delito de feminicidio. Esta se implementó el 23 de noviembre de 2016 en los municipios de Boca del Río, Coatzacoalcos, Córdoba, Las Choapas, Martínez de la Torre, Minatitlán, Orizaba, Poza Rica, Tuxpan, Veracruz y Xalapa. El ordenamiento federal también recomendó "acciones específicas" en los municipios con población indígena, aunque no se delimitó cuáles eran estos ni describió en qué consistían tales acciones específicas.

Una pregunta recurrente entre diferentes sectores de la ciudadanía es si la declaratoria ha incidido en un cambio cuantitativo y cualitativo. (Ver tabla 1)

En la tabla uno se concentran los datos referentes a tipos y modalidades de violencia, desapariciones, feminicidios y homicidios de mu-

10 Al sumar el número de mujeres que se ubican en estos rangos de edad, nos encontramos con 694 víctimas mortales. Se infiere que las edades del total de víctimas no se pudo establecer debido a las condiciones que presentaban los cadáveres o porque se omitió el dato en el expediente de registro, entre otros factores.

11 Estas cifras contrastan de manera dramática con lo registrado por la organización CIDEM, la cual estableció que para 2012 hubo 95 feminicidios y para 2013 hubo un total de 92. Asimismo, el proyecto universitario Asesinatos de mujeres y niñas por razón de género. Feminicidio en la entidad veracruzana, estableció que en 2014 tuvieron lugar 74 eventos de esta naturaleza; lo cual nos arroja un total de 261 feminicidios en tres años.

12 Equifonía Colectivo por la Ciudadanía, Autonomía y Libertad de las Mujeres, fue la organización firmante de la solicitud; Kalli Luz Marina, Colectivo Akelarre, Colectivo Feminista, Buscamos a Nuestras Hijas, Salud y Género, Agrupación de Derechos Humanos Xochitépetl, Red Unidos por los Derechos Humanos, Asesoría y Servicios Rurales, Integración, Lucha y Transformación fueron las organizaciones solicitantes que respaldaron la solicitud. 
jeres, así como la sumatoria de estos dos rubros que se reflejan en la última columna. La información ahí presentada corresponde al periodo que va del 1 de enero al 30 de junio de 2016. También nos muestra los municipios veracruzanos con mayor incidencia. La primer columna muestra que en el lapso referido tuvieron lugar 538 agresiones contra mujeres. Coatzacoalcos fue el municipio que presentó más casos, seguido por San Andrés Tuxtla, Acayucan, Veracruz y Xalapa. En lo referente a desapariciones, hubo 171 reportes, sobresaliendo Xalapa con el mayor número.

En los primeros seis meses de 2016 se registraron 63 eventos que reunían las características para ser considerados como feminicidios.
Es de llamar la atención que para ese momento del año, Tihuatlán, Papantla, Veracruz y Xalapa encabezaban a los municipios con más eventos de este tipo, con cinco casos cada uno. En la columna de homicidios de mujeres son 19 los registros correspondientes, siendo Córdoba el que concentraba el mayor número de mujeres víctimas en la entidad.

Hasta el 30 de junio de 2016 había 82 mujeres asesinadas, siendo el municipio que alberga a la capital del estado, el que encabezaba la lista. Si tomamos en cuenta que en 2014 habían tenido lugar 74 feminicidios y en 2015 se registraron 99, el primer semestre de este año era alarmante y la Alerta de Violencia de Género aún no se decretaba.

\section{Tabla1. Primer semestre de 2016}

\begin{tabular}{|c|c|c|c|c|}
\hline $\begin{array}{c}\text { Tipos y modalidades } \\
\text { de violencia contra } \\
\text { mujeres }\end{array}$ & Desapariciones & Feminicidios & $\begin{array}{c}\text { Homicidios de } \\
\text { mujeres }\end{array}$ & $\begin{array}{c}\text { Asesinatos de } \\
\text { mujeres }\end{array}$ \\
\hline $\begin{array}{c}\text { Total } \\
538\end{array}$ & $\begin{array}{c}\text { Total } \\
171\end{array}$ & $\begin{array}{c}\text { Total } \\
63\end{array}$ & $\begin{array}{c}\text { Total } \\
19\end{array}$ & $\begin{array}{c}\text { Total } \\
82\end{array}$ \\
\hline Municipios & Municipios & Municipios & Municipios & Municipios \\
\hline $\begin{array}{c}\text { Coatzacoalcos } \\
(155)\end{array}$ & $\begin{array}{c}\text { Xalapa } \\
(40)\end{array}$ & $\begin{array}{c}\text { Tihuatlán } \\
(5)\end{array}$ & $\begin{array}{c}\text { Córdoba } \\
(5)\end{array}$ & $\begin{array}{c}\text { Xalapa } \\
\text { (9) }\end{array}$ \\
\hline $\begin{array}{c}\text { San Andrés Tuxtla } \\
(111)\end{array}$ & $\begin{array}{c}\text { Coatzacoalcos } \\
(29)\end{array}$ & $\begin{array}{c}\text { Papantla } \\
(5)\end{array}$ & $\begin{array}{c}\text { Xalapa } \\
(4)\end{array}$ & $\begin{array}{c}\text { Veracruz } \\
\text { (8) }\end{array}$ \\
\hline Acayucan & $\begin{array}{c}\text { Veracruz } \\
(70)\end{array}$ & $\begin{array}{c}\text { Veracruz } \\
(25)\end{array}$ & $\begin{array}{c}\text { Orizaba } \\
(3)\end{array}$ & $\begin{array}{c}\text { Córdoba } \\
\text { (5) }\end{array}$ \\
\hline $\begin{array}{c}\text { Veracruz } \\
(58)\end{array}$ & $\begin{array}{c}\text { Minatitlán } \\
(24)\end{array}$ & $\begin{array}{c}\text { Xalapa } \\
(5)\end{array}$ & $\begin{array}{c}\text { Veracruz } \\
(3)\end{array}$ & $\begin{array}{c}\text { Tihuatlán } \\
\text { (5) }\end{array}$ \\
\hline Xalapa & Acayucan & San Andrés & $\begin{array}{c}\text { Gutiérrez Zamora } \\
(2)\end{array}$ & $\begin{array}{c}\text { Papantla } \\
\text { (5) }\end{array}$ \\
\hline (51) & $(15)$ & $(4)$ & & \\
\hline
\end{tabular}

Fuente: Asesinatos de mujeres y niñas por razón de género. Feminicidio en la entidad veracruzana. Facultad de Antropología, Universidad Veracruzana.

13 Para la tipificación del delito de feminicidio en el estado de Veracruz, el 11 de julio de 2012 se estableció el "Protocolo de diligencias básicas a seguir por el ministerio público en la investigación del delito de violencia feminicida” (Gobierno del estado de Veracruz, 2012). Establecía que para que los asesinatos de mujeres sean considerados como feminicidios, deben de presentar al menos alguna de las siguientes hipótesis: 1) que exista o haya existido una relación entre el activo y la víctima, una relación de parentesco por consanguinidad o afinidad, de matrimonio, concubinato, noviazgo o cualquier otra relación de hecho o amistad; 2) que exista o haya existido una relación laboral, escolar o que implique confianza, subordinación o superioridad, entre el activo y la víctima; 3) que la víctima presente signos de violencia sexual de cualquier tipo; 4) que presente lesiones infamantes, degradantes o mutilaciones previas a la muerte, o marcas infamantes, degradantes o mutilaciones sobre el cadáver; 5) que hayan existido amenazas, acoso o lesiones del sujeto activo en contra de la víctima; 6) que el cuerpo de la víctima sea expuesto o arrojado en un lugar público; 7) que la víctima haya sido incomunicada (Ibid). 
Sin embargo, lo acontecido en el primer semestre de 2017 no tendría precedentes, pues superaría por mucho a los años anteriores.

129 veracruzanas fueron asesinadas del 1 de enero al 30 de junio de 2017, siendo Poza Rica y Córdoba los que albergaban más casos. Un total de 89 feminicidios se presentaron. Nuevamente Poza Rica encabezaría la lista de los municipios con mayores eventos de este tipo, junto con Minatitlán y Veracruz. Por otra parte, podemos observar un registro de $40 \mathrm{mu}$ jeres víctimas de homicidio. Córdoba y Coatzacoalcos concentrarían siete casos cada uno, situación por la que fueron los municipios con más hechos de esta naturaleza.

En contraste con el año anterior, 2017 presentó un número ligeramente menor de desapariciones, las cuales ascendieron a 133. La mayoría se ubicaría en el municipio de Coatzacoalcos. También fueron menores los reportes de violencia al registrarse 499. De estos, 108 acontecieron en el municipio de San Andrés Tuxtla.

Se reitera que la información aquí presentada corresponde a estimaciones derivadas de las noticias que diariamente difunden los medios de comunicación y de la información que comparten las familias de las víctimas en un acto desesperado por denunciar los casos y exigir justicia. Los datos oficiales que alberga la Fiscalía General del Estado no se han dado a conocer a la población bajo ningún formato.

Por tanto, desconocemos el número de sentencias por feminicidio u homicidio de mujeres, o la posible conexión entre el tráfico de mujeres y las desapariciones forzadas de las mismas o si las jóvenes que "se van con el novio" en realidad son víctimas de enganchadores profesionales que las internan en el mundo del trabajo sexual forzado.

Tabla 2. Primer semestre de 2017

\begin{tabular}{|c|c|c|c|c|}
\hline $\begin{array}{c}\text { Tipos y modalidades } \\
\text { de violencia }\end{array}$ & $\begin{array}{l}\text { Desapariciones de } \\
\text { mujeres y niñas }\end{array}$ & Feminicidios & $\begin{array}{l}\text { Homicidios de } \\
\text { mujeres }\end{array}$ & $\begin{array}{c}\text { Asesinatos de } \\
\text { mujeres }\end{array}$ \\
\hline $\begin{array}{l}\text { Total } \\
499\end{array}$ & $\begin{array}{l}\text { Total } \\
133\end{array}$ & $\begin{array}{c}\text { Total } \\
89\end{array}$ & $\begin{array}{c}\text { Total } \\
40\end{array}$ & $\begin{array}{c}\text { Total } \\
129\end{array}$ \\
\hline Municipios & Municipios & Municipios & Municipios & Municipios \\
\hline $\begin{array}{l}\text { San Andrés Tuxtla } \\
\text { (108) }\end{array}$ & $\begin{array}{c}\text { Coatzacoalcos } \\
\text { (18) }\end{array}$ & $\begin{array}{l}\text { Poza Rica } \\
\text { (6) }\end{array}$ & $\begin{array}{l}\text { Córdoba } \\
\text { (7) }\end{array}$ & $\begin{array}{l}\text { Poza Rica } \\
\text { (9) }\end{array}$ \\
\hline $\begin{array}{c}\text { Coatzacoalcos } \\
\text { (99) }\end{array}$ & $\begin{array}{l}\text { Acayucan } \\
\quad(10)\end{array}$ & $\begin{array}{l}\text { Minatitlán } \\
\text { (6) }\end{array}$ & $\begin{array}{l}\text { Coatzacoalcos } \\
\text { (7) }\end{array}$ & $\begin{array}{l}\text { Córdoba } \\
\text { (9) }\end{array}$ \\
\hline $\begin{array}{l}\text { Acayucan } \\
\quad(59)\end{array}$ & $\begin{array}{l}\text { Poza Rica } \\
\text { (8) }\end{array}$ & $\begin{array}{l}\text { Veracruz } \\
\text { (6) }\end{array}$ & $\begin{array}{l}\text { Acayucan } \\
\quad(4)\end{array}$ & $\begin{array}{l}\text { Minatitlán } \\
\text { (8) }\end{array}$ \\
\hline $\begin{array}{l}\text { Poza Rica } \\
\text { (18) }\end{array}$ & $\begin{array}{l}\text { Papantla } \\
\text { (6) }\end{array}$ & $\begin{array}{l}\text { Ixtaczoquitlán } \\
\text { (4) }\end{array}$ & $\begin{array}{l}\text { Poza Rica } \\
\text { (3) }\end{array}$ & $\begin{array}{c}\text { Coatzacoalcos } \\
\text { (7) }\end{array}$ \\
\hline $\begin{array}{l}\text { Veracruz } \\
\quad(14)\end{array}$ & $\begin{array}{l}\text { Las Choapas } \\
\text { (6) }\end{array}$ & $\begin{array}{l}\text { Sayula } \\
\text { (4) }\end{array}$ & $\begin{array}{l}\text { Coatzintla } \\
\text { (2) }\end{array}$ & $\begin{array}{l}\text { Veracruz } \\
\quad(6)\end{array}$ \\
\hline $\begin{array}{l}\text { Xalapa } \\
\text { (8) }\end{array}$ & $\begin{array}{l}\text { Xalapa } \\
(5)\end{array}$ & $\begin{array}{l}\text { Alvarado } \\
\text { (3) }\end{array}$ & $\begin{array}{l}\text { Orizaba } \\
\text { (2) }\end{array}$ & $\begin{array}{l}\text { Acayucan } \\
\text { (4) }\end{array}$ \\
\hline $\begin{array}{l}\text { Tuxpan } \\
(7)\end{array}$ & $\begin{array}{l}\text { Minatitlán } \\
\text { (5) }\end{array}$ & $\begin{array}{l}\text { Córdoba } \\
\text { (2) }\end{array}$ & $\begin{array}{l}\text { Cuitláhuac } \\
\text { (2) }\end{array}$ & $\begin{array}{c}\text { Ixtaczoquitlán } \\
\text { (4) }\end{array}$ \\
\hline $\begin{array}{l}\text { Córdoba } \\
\text { (7) }\end{array}$ & $\begin{array}{l}\text { Veracruz } \\
\text { (5) }\end{array}$ & $\begin{array}{l}\text { Tuxpan } \\
\text { (2) }\end{array}$ & $\begin{array}{l}\text { Minatitlán } \\
\text { (2) }\end{array}$ & $\begin{array}{l}\text { Sayula } \\
\text { (4) }\end{array}$ \\
\hline
\end{tabular}

Fuente: Asesinatos de mujeres y niñas por razón de género. Feminicidio en la entidad veracruzana. Facultad de Antropología, Universidad Veracruzana. 


\section{Conclusiones}

2017 ha sido un año bastante violento para la población veracruzana en general y para las mujeres en particular. Pese a la declaratoria de Alerta de Violencia de Género implementada por el gobierno federal, los asesinatos de mujeres se siguen cometiendo, solo que ahora en mayor número, aunque con el mismo grado de impunidad.

La población en general desconoce las acciones derivadas del trabajo coordinado entre gobierno del estado y gobierno federal para atender las exigencias de este mecanismo. Sin embargo, el elevado número de feminicidios acontecidos en el marco de la Alerta nos hace reflexionar en torno al alcance de dichas acciones. La situación es sumamente preocupante e incierta.

En tanto, no debemos de perder de vista que el escenario anteriormente presentado permite conocer que existen:

a) Condiciones estructurales que favorecen la violencia y asesinatos contra las mujeres.

b) A partir de lo anterior se fortalece un sistema de dominación masculina que recrudece los mecanismos de género que violentan y asesinan a las mujeres

c) Fortalecimiento del crimen organizado y sus negocios ilícitos. Uno de los más redituables es aquél que fortalecen el tráfico de mujeres para el trabajo sexual forzado

d) El grado de impunidad y la indiferencia del poder judicial ante los delitos cometidos contra veracruzanas alienta y detona el incremento de las violencias contra las mujeres.

Este documento constituye una modesta aproximación al tema que tiene la intención de hacer palpable que, a pesar de los instrumentos legales estatales y nacionales para el avance de las mujeres, los mecanismos para salvaguardar sus vidas y la apertura de las instituciones dedicadas a mejorar sus condiciones y empoderarlas, por parte del Estado hay un brutal des- precio por la vida de las ciudadanas cuando se trata de impactar con acciones cualitativamente decisivas que garanticen el bienestar de las veracruzanas.

\section{Referencias}

Almerás, Diane y Coral Calderón Magaña (coordinadoras) (2012). Si no se cuenta, no cuenta. Información sobre la violencia contra las mujeres. Santiago de Chile: Comisión Económica para América Latina y el Caribe (CEPAL).

Comisión Económica para América Latina y el Caribe (CEPAL) (2014). Informe anual 2013-2014. El enfrentamiento de la violencia contra las mujeres en América Latina y el Caribe. Santiago de Chile: CEPAL.

Comisión Nacional para Prevenir y Erradicar la Violencia contra las Mujeres (CONAVIM) (2015). Informe del Grupo de Trabajo conformado para estudiar la Solicitud de Alerta de Violencia de Género contra las Mujeres en el estado de Veracruz. CONAVIM. Portal oficial consultado el 13 de enero de 2017 en:

https://www.gob.mx/cms/uploads/attachment/ file/53138/Inf_AVGM_Veracruz.pdf

Consejo Nacional de Evaluación de la Política de Desarrollo Social (CONEVAL) (2017a). Carencias sociales 2015 y su comparativo con la serie 2010-2014. Veracruz. Portal oficial consultado el 11 de agosto de 2017 en:

http://www.coneval.org.mx/coordinacion/entidades/ Veracruz/Paginas/carencias-sociales20102015.aspx

CONEVAL (2017b). "CONEVAL da a conocer la evolución de las carencias sociales 2015 y su comparativo con la serie 2010-2014". Comunicado de prensa, núm. 03, 20 pp. Portal oficial consultado el 11 de agosto de 2017 en: http://www.coneval.org.mx/SalaPrensa/Comunicadosprensa/Documents/COMUNICADO-03-Evolucion-Carencias-Sociales-2015.pdf

CONEVAL (2014). "La pobreza agudiza la desigualdad de género: CONEVAL”, . Comunicado de prensa, núm. 003, 6 pp. Portal oficial consultado el 11 de agosto de 2017 en: http://www.coneval.org.mx/Informes/COMUNICADOS_DE_PRENSA/2014/COMUNICADO_003_ESTUDIO_POBREZA_Y_GENERO_2008_2012.pdf

Gobierno del estado de Veracruz (2012). Gaceta oficial, tomo CLXXXVI. Núm. ext. 228, 11 de julio de 2012.

Instituto Nacional de las Mujeres (INMUJERES) (2009). Ley General de Acceso de las Mujeres a una Vida Libre de Violencia. ¡Conócela!, México: INMUJERES. Consul- 
tada el 9 de agosto en:

https://www.gob.mx/cms/uploads/attachment/ file/178863/ley-general-acceso-mujeres-vida-libre-violencia.pdf

Organización de las Naciones Unidas (1993). "Declaración sobre la eliminación de la violencia contra la mujer". Resolución 48/104 del 20 de diciembre. Consultado el 9 de agosto de 2017 en:

http://www.ordenjuridico.gob.mx/TratInt/Derechos\%20Humanos/INST\%2018.pdf

Programa de las Naciones Unidas para el Desarrollo (PNUD) (2015). Índice de Desarrollo Humano para las entidades federativas, México 2015. Avance continuo, diferencias persistentes. México: PNUD.

PNUD, INMUJERES, ONU Mujeres (2016). Desarrollo humano y violencia contra las mujeres en México. PNUD, INMUJERES, ONU Mujeres. Consultado el 10 de agosto de 2017 en:

http://www.mx.undp.org/content/mexico/es/home/ library/poverty/desarrollo-humano-y-violencia-contra-las-mujeres-en-mexico/

Rodríguez Herrero, Hipólito y Gilberto Cházaro (2013). "Desigualdad e inseguridad en Veracruz" en Alberto J. Olvera Rivera, Alfredo Zavaleta Betancourt y Víctor Manuel Andrade Guevara (coordinadores). Violencia, inseguridad y justicia en Veracruz. Xalapa: Universidad Veracruzana. Pp. 21-51. 